\title{
A General Composition Theorem for Secure Reactive Systems
}

\author{
Michael Backes, Birgit Pfitzmann, and Michael Waidner \\ IBM Zurich Research Lab \\ $\{$ mbc, bpf, wmi $\} @$ zurich.ibm.com
}

\begin{abstract}
We consider compositional properties of reactive systems that are secure in a cryptographic sense. We follow the well-known simulatability approach of modern cryptography, i.e., the specification is an ideal system and a real system should in some sense simulate this ideal one. We show that if a system consists of a polynomial number of arbitrary ideal subsystems such that each of them has a secure implementation in the sense of blackbox simulatability, then one can securely replace all ideal subsystems with their respective secure counterparts without destroying the blackbox simulatability relation. We further prove our theorem for universal simulatability by showing that blackbox simulatability implies universal simulatability under reasonable assumptions. We show all our results with concrete security.
\end{abstract}

\section{Introduction}

In recent times, the analysis of cryptographic protocols has been getting more and more attention, and thus the demand for general frameworks for representing cryptographic protocols and their security requirements has been rising. To enable a cryptographically correct analysis of cryptographic protocols, such frameworks have to capture probabilistic behaviors, complexity-theoretically bounded adversaries as well as a reactive environment of the protocol, i.e., continuous interaction with users and an adversary, e.g., in many protocol runs. Clearly, such frameworks further have to be rigorously defined to avoid ambiguities and to enable convincing proofs. Moreover, it is highly desirable that such frameworks provide a link to formal methods, i.e., to tool-supported verification of cryptographic protocols. Tool support can minimize flaws, which occur quite often if the distributed-systems aspects of cryptographic protocols are analyzed by hand. One ingredient for this is that the model should contain an abstract machine model besides Turing machines. The model of Pfitzmann and Waidner [31] is suitable for all these requirements and we use it as a rigorous foundation of this work.

The model of [31] introduced a notion of security-preserving refinement, called reactive simulatability. This notion captures the idea of refinement that preserves not only integrity properties but also confidentiality properties. Intuitively it can be stated as follows, when applied to the relation between a real and an ideal system 1 Everything that can happen to users of the real system in the presence of an arbitrary adversary A' can also happen to the same users with the ideal system, where attack capabilities are

\footnotetext{
${ }^{1}$ Other terms are implementation and specification, or in special cases cryptographic and abstract system.
} 
usually much more restricted, in the presence of another adversary A. In particular, it comprises confidentiality because the notion of what happens to users, called their view, not only includes their in- and outputs to the system, but also their communication with the adversary. This includes whether the adversary can guess secrets of the users or partial information about them. As it is often desirable to impose further restrictions on how the adversary A against the ideal service is constructed, simulatability comes in different flavors. The two most prominent ones (besides general simulatability as described above, which does not impose any restriction on A) are universal simulatability, which states that $\mathrm{A}$ has to be independent of the actual users of the protocol, and the (seemingly) more restrictive notion of black-box simulatability, which states that A consists of the original adversary A' and a simulator that may only depend on the protocol itself.

One of the key results in the considered model is a composition theorem [31]. It states that if a larger system is designed based on a specification of a subsystem, and the implementation of the subsystem is later plugged in, the entire implementation of the larger system is as secure as its design in the same sense of reactive simulatability. This theorem (as well as its predecessor [30] for a synchronous reactive model) holds for all variants of simulatability (general, universal, and blackbox), but it is restricted to replacing one system. Obviously, a constant number of systems can then be replaced by applying the theorem multiple times.

In this work, we present a more comprehensive composition theorem for blackbox simulatability by showing that a polynomial number (in a security parameter) of arbitrary systems can be composed without destroying the simulatability relation. The proof relies on what is often called a "standard hybrid argument" as first used in [15]. We further show that universal simulatability implies black-box simulatability under reasonable assumptions. This is of independent interest, but it in particular allows us to prove our theorem also for universal simulatability. We show all our results with concrete security.

Related Literature. Simulatability was first sketched for secure multi-party function evaluation, i.e., for the computation of one output tuple from one tuple of secret inputs from each participant in [33] and defined (with different degrees of generality and rigorosity) in [14.627|9]. While composition theorems for special cases were proven in [6, 27], the first general composition theorem for non-reactive simulatability was proven in [9].

An important step towards compositionality results of reactive systems was taken in [1920], where the cryptographic security of specific systems was directly defined and verified using a formal language, the $\pi$-calculus, and security was expressed using observational equivalence. This notion is even stronger than reactive simulatability because the entire environment (corresponding to users and adversary together for reactive simulatability) must not be able to distinguish the implementation and the specification. Correspondingly, the concrete specifications used were not abstract; they essentially comprise the actual protocols including all cryptographic details. Composition was defined in the calculus by defining processes with "holes" for other processes, which then allows for composing a constant number of systems.

A reactive simulatability definition was first proposed (after some earlier sketches, in particular in [13.299]) in [16]. It is synchronous, covers a restricted class of protocols (straightline programs with restricted operators, in view of the constructive result of this 
paper), and simulatability is defined for the information-theoretic case only, where it can be done with a quantification over input sequences instead of active honest users.

The first composition theorem for reactive simulatability was given in [30] for a general synchronous reactive model, followed by essentially the same composition theorem [31] in the corresponding asynchronous model. Later than [31] but independently, another model of asynchronous reactive systems together with a composition theorem for reactive simulatability was developed in [10]. The theorem is specific for universal simulatability, but for this case it is more general than the ones in [30,31] since it additionally allows for securely composing a polynomial number of copies of an ideal service, which naturally correspond to different protocol instances in the real implementation. We stress that our composition theorem in this paper not only captures secure composition of a polynomial number of copies of one single ideal system but also of a polynomial number of truly arbitrary systems. However, our work was inspired by [10].

Besides considering composition as secure refinement, property-based composition has received interest in the literature: It considers the question whether systems that individually provide certain security properties still have these properties when they are run in parallel with other systems. For safety and liveness, general theories of this kind of compositionality exist [28 32,1], which are sufficient to reason about most functional system properties. However, many security properties are not safety and liveness properties, in particular confidentiality. Compositional information flow properties were first investigated in [23]. After that, much work has been devoted to identifying properties which are preserved under composition like, e.g., restrictiveness [23 24], forward correctability [18], or separability [25]. For certain security properties that are in general not preserved under composition, it is known how to restrict composition in order to preserve these properties [25 26]. More recent work concentrated on a uniform basis to reason about property-based composition [2211].

Somewhere between both notions of composition, so-called preservation theorems exist, which state that specific properties are preserved under (reactive) simulatability. Such theorems exist for integrity [2], transitive and non-transitive non-interference [3, 4], i.e., absence of information flow, and a class of liveness properties [5].

Outline. In Section 2 we review the model of reactive systems in asynchronous networks. Section 3 contains our composition theorem and its proof for black-box simulatability. In Section 4, we show that universal simulatability implies black-box simulatability and reasonable assumptions. In particular, this can be used to carry over our composition theorem for universal simulatability.

\section{Asynchronous Reactive Systems}

In this section, we review our model for secure reactive systems in an asynchronous network from [31]. Several definitions are only sketched whereas those that are important for understanding our results are given in full detail. All other details can be looked up in the original paper.

\subsection{General System Model}

Systems mainly consist of several interactive machines. Machines communicate via ports (local endpoints for different potential channels) and messages are strings over 


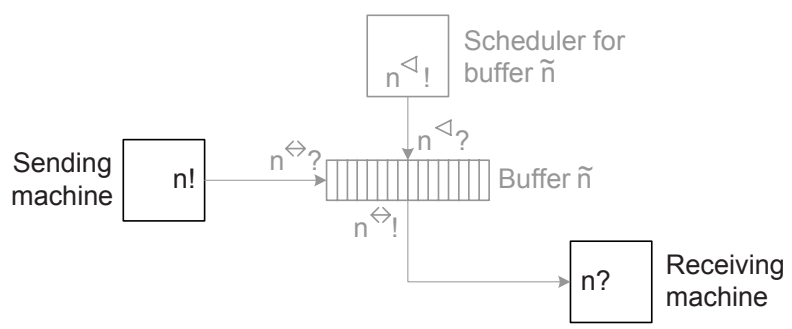

Fig. 1. Ports and buffers. Specifications only need to spell out the black part

an alphabet $\Sigma$. Inspired by the CSP-Notation [17], we write output and input ports as $\mathrm{q}$ ! and $\mathrm{q}$ ? respectively. As in similar models, channels are defined implicitly by naming convention (and not by a separate graph), that is port q! sends messages to q?. For asynchronous timing, a message is not immediately delivered to its recipient, but first stored in a special machine $\widetilde{q}$ called a buffer. If a machine wants to schedule the $i$-th message of buffer $\widetilde{\mathrm{q}}$, it must have the unique clock-out port $\mathrm{q}^{\triangleleft}$ !, and it sends $i$ at $\mathrm{q}^{\triangleleft}$ !, see Figure 1 The buffer then outputs and deletes its $i$-th message. For a port $p$, we write $p^{c}$ to denote the port which it connects to according to Figure 1, i.e., $\mathrm{q} !^{c}=\mathrm{q} \leftrightarrow$ ?, $\mathrm{q}^{\leftrightarrow} !^{c}=\mathrm{q}$ ?, $\mathrm{q}^{\triangleleft} !^{c}=\mathrm{q}^{\triangleleft}$ ? and vice versa. The in- and output ports in a port set or port sequence $P$ are denoted in $(P)$ and out $(P)$.

Our primary machine model is probabilistic state-transition machines, similar to probabilistic I/O automata as in Lynch [21] (and also essentially in [6/27]). If a machine is switched, it receives an input tuple at its input ports and performs its transition function. This yields a new state and an output tuple in the deterministic case, or a finite distribution over such pairs in the probabilistic case. Moreover, each machine has a function bounding the length of the considered inputs; this allows flexible time bounds independent of the environment.

Definition 1. (Machines) A machine is a tuple

$$
\mathrm{M}=\left(\text { name }_{\mathrm{M}}, \text { Ports }_{\mathrm{M}}, \text { States }_{\mathrm{M}}, \delta_{\mathrm{M}}, l_{\mathrm{M}}, \text { Ini }_{\mathrm{M}}, \text { Fin }_{\mathrm{M}}\right)
$$

of a name name na $_{\mathrm{M}} \in \Sigma^{+}$, a finite sequence Ports $s_{\mathrm{M}}$ of ports, a set States $\mathrm{M} \subseteq \Sigma^{*}$ of states, a probabilistic state-transition function $\delta_{\mathrm{M}}$, a length function $l_{\mathrm{M}}:$ States $\mathrm{M} \rightarrow$ $(\mathbb{N} \cup\{\infty\})^{\mid \operatorname{in}\left(\text { Ports }_{\mathrm{M}}\right) \mid}$, and sets Ini $_{\mathrm{M}}$, Fin $_{\mathrm{M}} \subseteq$ States $_{\mathrm{M}}$ of initial and final states. Its input set is $\mathcal{I}_{\mathrm{M}}:=\left(\Sigma^{*}\right)^{\left|\operatorname{in}\left(\operatorname{Ports}_{\mathrm{M}}\right)\right|}$; the $i$-th element of an input tuple denotes the input at the $i$-th in-port. Its output set is $\mathcal{O}_{\mathrm{M}}:=\left(\Sigma^{*}\right)^{\mid \text {out }\left(\text { Port }_{\mathrm{M}}\right) \mid}$. The empty word, $\epsilon$, denotes no in- or output at a port. $\delta_{\mathrm{M}}$ probabilistically maps each pair $(s, I) \in$ States $_{\mathrm{M}} \times \mathcal{I}_{\mathrm{M}}$ to a pair $\left(s^{\prime}, O\right) \in$ States $_{\mathrm{M}} \times \mathcal{O}_{\mathrm{M}}$.

Two restrictions apply to $\delta_{\mathrm{M}}$ : Every output distribution has to be finite and if $I=$ $(\epsilon, \ldots, \epsilon)$, then $\delta_{\mathrm{M}}(s, I)=(s,(\epsilon, \ldots, \epsilon))$. Inputs are ignored beyond the length bounds, i.e., $\delta_{\mathrm{M}}(s, I)=\delta_{\mathrm{M}}\left(s, I\left\lceil_{l_{\mathrm{M}}(s)}\right)\right.$ for all $I \in \mathcal{I}_{\mathrm{M}}$, where $r\left\lceil_{l}\right.$ for $l \in \mathbb{N}, r \in \Sigma^{*}$ denotes the $l$ symbol prefix, and the notation is lifted to tuples. We further demand $l_{\mathrm{M}}(s)=(0, \ldots, 0)$ for every $s \in$ Fin $_{\mathrm{M}}$.

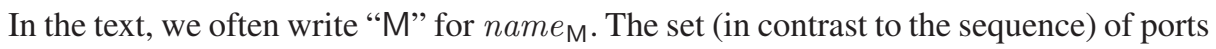
of a machine $M$ is denoted by ports $(M)$, and similar for sets of machines. 
A collection $\hat{C}$ of machines is a set of machines with pairwise different machine names and disjoint sets of ports. The completion $[\hat{C}]$ of a collection $\hat{C}$ is the union of all machines of $\hat{C}$ and the buffers needed for every channel. A port of a collection is called free if its connecting port is not in the collection. These ports will be connected to the users and the adversary. The free ports of a completion $[\hat{C}]$ are denoted as free $([\hat{C}])$. A collection $\hat{C}$ is called closed if its completion $[\hat{C}]$ has no free ports except a special master clock-in port $\mathrm{clk}^{\triangleleft}$ ?.

A closed collection represents a "runnable" system and a probability space of runs (sometimes called traces or executions) is defined for it. Machines switch sequentially, i.e., we have exactly one active machine $\mathrm{M}$ at any time. If this machine has clock outports, it can select the next message to be delivered by scheduling a buffer via one of these clock out-ports. If the buffer contains a message at the selected position, it delivers this message, and the receiving machine is the next active machine. If $\mathrm{M}$ tries to schedule multiple messages, only one is taken, and if it schedules none or the message does not exist, the master scheduler $\mathrm{X}$ becomes active. Formally, runs are defined as follows.

Definition 2. (Runs and Views) Let $\hat{C}$ be a closed collection with master scheduler $\mathrm{X}$. Runs and their probability spaces are defined inductively by the following algorithm for each tuple $i n i \in \times_{\mathrm{M} \in \hat{C}}$ Ini $_{\mathrm{M}}$ of initial states. The algorithm maintains variables for the state of each machine and treats each port as a variable over $\Sigma^{*}$, initialized with $\epsilon$ except for $\mathrm{clk}^{\triangleleft} ?:=1$. It further maintains a variable $\mathrm{M}_{\mathrm{CS}}$ ("current scheduler") over machine names, initially $\mathrm{M}_{\mathrm{CS}}:=\mathrm{X}$, for the currently active non-buffer machine, and a variable $r$ for the resulting run, an initially empty list. The algorithm has five phases. Probabilistic choices only occur in Phase 1

1. Switch current scheduler: Switch the current machine $\mathrm{M}_{\mathrm{CS}}$, i.e., set $\left(s^{\prime}, O\right) \leftarrow$ $\delta_{\mathrm{M}_{\mathrm{CS}}}(s, I)$ for its current state $s$ and in-port values $I$. Then assign $\epsilon$ to all in-ports of $\mathrm{M}_{\mathrm{CS}}$.

2. Termination: If $\mathrm{X}$ is in a final state, the run stops. (As $\mathrm{X}$ made no outputs in this case, this only prevents repeated master clock inputs.)

3. Store outputs: For each simple out-port o! of $\mathrm{M}_{\mathrm{CS}}$ with $\mathrm{o} ! \neq \epsilon$, in their given order, switch buffer $\widetilde{0}$ with input $\mathrm{o}^{\leftrightarrow} ?:=\mathrm{o}$ !. Then assign $\epsilon$ to these ports $\mathrm{o}$ ! and $\mathrm{\circ} \leftrightarrow ?$ ?

4. Clean up scheduling: If at least one clock out-port of $\mathrm{M}_{\mathrm{CS}}$ has a value $\neq \epsilon$, let $\mathrm{n}^{\triangleleft}$ ! denote the first such port and assign $\epsilon$ to the others. Otherwise let $\mathrm{clk}^{\triangleleft} ?:=1$ and $\mathrm{M}_{\mathrm{CS}}:=\mathrm{X}$ and go to Phase 1 .

5. Deliver scheduled message: Switch buffer $\widetilde{\mathrm{n}}$ with input $\mathrm{n}^{\triangleleft}$ ? $:=\mathrm{n}^{\triangleleft}$ !, set $\mathrm{n}$ ? $:=\mathrm{n} \leftrightarrow$ ! and then assign $\epsilon$ to all ports of $\widetilde{\mathrm{n}}$ and to $\mathrm{n}^{\triangleleft}$ !. If $\mathrm{n}$ ? $=\epsilon$ let $\mathrm{clk}^{\triangleleft}$ ? $:=1$ and $\mathrm{M}_{\mathrm{CS}}:=\mathrm{X}$. Else let $\mathrm{M}_{\mathrm{CS}}:=\mathrm{M}^{\prime}$ for the unique machine $\mathrm{M}^{\prime}$ with $\mathrm{n} ? \in \operatorname{ports}\left(\mathrm{M}^{\prime}\right)$. Go to Phase 1

Whenever a machine (this may be a buffer) $\mathrm{M}$ switches from $(s, I)$ to $\left(s^{\prime}, O\right)$, we add a step $\left(\right.$ name $\left._{\mathrm{M}}, s, I, s^{\prime}, O\right)$ to the run $r$ with the following two restrictions. First, we cut each input according to the respective length function, i.e., we replace $I$ by $I^{\prime}:=I\left\lceil_{l_{\mathrm{M}}(s)}\right.$. Secondly, we do not add the step to the run if $I^{\prime}=(\epsilon, \ldots, \epsilon)$, i.e., if nothing happens in reality. This gives a random variable run $_{\hat{C}, \text { ini }}$ for each tuple ini $\in \times_{\mathrm{M} \in \hat{C}}$ of initial states, and similarly for $l_{\text {-step prefixes }}$ run $\hat{C}_{\hat{C}, \text { ini, }}{ }^{*}$

The view of a subset $\hat{M} \subseteq \hat{C}$ of machines in a run $r$ is the subsequence of $r$ consisting of those steps where a machine of $\hat{M}$ switches. This gives a random variable view $\hat{C}_{\text {, ini }}(\hat{M})$ for each tuple ini of initial states, and similarly for l-step prefixes 
view $_{\hat{C}, \text { ini,l }}(\hat{M})$ of the view. For a singleton $\hat{M}=\{\mathrm{H}\}$ we write view $\hat{C}_{\text {, ini }}(\mathrm{H})$ for view $_{\hat{C}_{\text {,ini }}}(\{\mathrm{H}\})$.

\subsection{Security-Specific System Model}

We now define specific collections for security purposes. We start with the definition of structures. Intuitively, these are the machines that execute a security protocol. They have a distinguished set of service ports. This is a subset of the free ports where, intuitively, a certain service is guaranteed, while remaining free ports are meant only for the adversary. Typical examples of inputs at service ports are "send message $m$ to participant $i d$ " for a message transmission system or "pay amount $x$ to participant $i d$ " for a payment system, while typical non-service ports are those of insecure network connections in a real system. For cryptographic purposes, the initial state of all machines in a structure is a security parameter $k$ in unary representation.

Definition 3. (Structures and Service Ports) A structure is a pair struc $=(\hat{M}, S)$ where $\hat{M}$ is a collection of simple machines (i.e., with only normal in- and out-ports and clock out-ports) with $\{1\}^{*} \subseteq \operatorname{Ini}_{\mathrm{M}}$ for all $\mathrm{M} \in \hat{M}$, and $S \subseteq$ free $([\hat{M}])$. The set $S$ is called service ports.

Forbidden ports for users of a structure are those that clash with port names of given machines and those that would link the user to a non-service port.

Definition 4. (Forbidden Ports) For a structure $(\hat{M}, S)$ let $\bar{S}_{\hat{M}}:=$ free $([\hat{M}]) \backslash S$. We call forb $(\hat{M}, S):=\operatorname{ports}(\hat{M}) \cup \bar{S}_{\hat{M}}^{c}$ the forbidden ports.

A system is a set of structures. The idea behind systems is that there may be different actual structures depending on the set of actually malicious participants.

Definition 5. (Systems) A system Sys is a set of structures.

A structure can be complemented to a configuration by adding a user machine and an adversary machine. The user is restricted to connecting to the service ports. The adversary closes the collection, i.e., it connects to the remaining service ports, the other free ports $\bar{S}_{\hat{M}}$ of the collection, and the free ports of the user. Thus, user and adversary can interact, e.g., for modeling active attacks.

Definition 6. (Configurations) $A$ configuration of a structure $(\hat{M}, S)$ is a tuple conf $=$ $(\hat{M}, S, \mathrm{H}, \mathrm{A})$ where

- $\mathrm{H}$ is a machine called user with ports $(\mathrm{H}) \cap \operatorname{forb}(\hat{M}, S)=\emptyset$ and $\{1\}^{*} \subseteq \operatorname{Ini}_{\mathrm{H}}$,

- A is a machine called adversary with $\{1\}^{*} \subseteq \operatorname{Ini}_{\mathrm{A}}$,

- and the completion $\hat{C}:=[\hat{M} \cup\{\mathrm{H}, \mathrm{A}\}]$ is a closed collection.

The set of configurations of $(\hat{M}, S)$ is written $\operatorname{Conf}(\hat{M}, S)$. The notation $\operatorname{Conf}()$ is lifted to sets of structures, i.e., systems. We write conf. $\hat{M}$ for conf $[1]$ (component selection function) and similarly conf.S, conf. $\mathrm{H}$, and conf.A, and conf.struc for conf $[1,2] . \diamond$ 


\subsection{Parametrized Systems}

In many typical systems, the structures only depend on the trust model, but not on the security parameter $k$. In a parametrized system this is different. Hence such a system is partitioned into different subsystems for different values of $k$. "Normal" systems can naturally be identified with parametrized systems where all subsystems are equal.

Definition 7. (Parametrized Systems) A parametrized system is a system Sys together with a partitioning $\left(S y s_{k}\right)_{k \in \mathbb{N}}$, i.e., the elements Sys $s_{k}$ are pairwise disjoint systems with Sys $=\bigcup_{k \in \mathbb{N}} S y s_{k}$. In slight abuse of notation we also call the sequence of partitions Sys, and if the system is called Sys, the notation Sys ${ }_{k}$ always refers to the $k$-th element in the partition sequence.

$A$ bounding function for a parametrized system is a function $P$ such that for all $k \in \mathbb{N}$ and $(\hat{M}, S) \in$ Sys $_{k}$ we have $|\hat{M}| \leq P(k)$ and the runtime of every $\mathrm{M} \in \hat{M}$ on initial input $1^{k}$ is bounded by $P(k)$ in the sense of circuit complexity (more precisely, circuit size). A parametrized system is polynomial-time if it has a polynomial bounding function.

Circuit complexity, i.e., non-uniform complexity, is natural for this definition because one can consider every machine $\mathrm{M}$, used only for security parameter $k$, as a separate circuit.As we want to bound the overall runtime of a machine with respect to its initial input length, just as in the uniform case, this can be defined by one normal non-cyclic circuit for each machine. Meaningful uniform complexity for such a definition requires a universal machine that simulates all these structures, and a generation algorithm for structures. However, our results are reductions with concrete security (as first introduced as a general concept with special notation in [8] ), and usable for a wide range of complexity measures. In those reductions we actually work with Turing complexity because it is defined in full detail for our interacting machines.

A parametrized system considers the potentially used subsystems as potentially available from the start. This is also implicitly the case in [10] because although a subsystem is said to be generated there, it springs up magically in distributed locations by this operation. This means that all the connections must be assumed to be predefined. A truly dynamic system would need to distribute port or machine names of new machines, like the $\pi$-calculus does. We do not see any specific reason while our theorem should not hold for this case but it would require a rigorous definition first.

We now define user and adversary of a parametrized system.

Definition 8. (User and Adversary of a Parametrized System) A user and an adversary of a parametrized system Sys are families $\left(\mathrm{H}_{\text {struc }}\right)_{\text {struc } \in \text { Sys }},\left(\mathrm{A}_{\text {struc }}\right)_{\text {struc } \in \text { Sys }}$ such that $\left(\hat{M}, S, \mathrm{H}_{(\hat{M}, S)}, \mathrm{A}_{(\hat{M}, S)}\right) \in \operatorname{Conf}($ Sys $)$ for all $(\hat{M}, S) \in$ Sys.

To reason about the complexity of users and adversaries, or more generally families of machines, we define the parametrized complexity.

Definition 9. (Parametrized Complexity) Let $X=\bigcup_{k \in \mathbb{N}} X_{k}$ be a partitioned index set (with the same conventions as for systems) and let $A=\left(A_{x}\right)_{x \in X}$ be a family of machines with $\{1\}^{*} \subseteq$ Ini $_{A_{x}}$ for every $x \in X$. We say that $A$ is of complexity $t: \mathbb{N} \rightarrow \mathbb{N}$ iffor all $x \in X_{k}$, the runtime of $A_{x}$ on initial input $1^{k}$ is bounded by $t(k)$ in the sense of circuit complexity. We sometimes write $t_{A}$ for "the" complexity of $A$. 


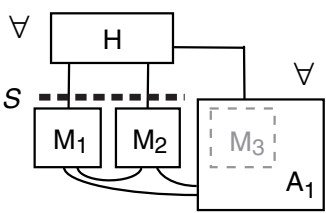

Real configuration

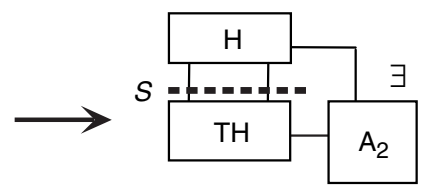

Ideal configuration

Fig. 2. Example of simulatability. The view of $\mathrm{H}$ is compared.

\subsection{Defining Security with Simulatability}

Reactive simulatability essentially means that whatever might happen to an honest user in a real system $S y s_{1}$ can also happen in an ideal system $S y s_{2}$. More precisely, for every configuration $\operatorname{conf}_{1}$ of $S y s_{1}$, there exists a configuration conf $_{2}$ of $S y s_{2}$ with the same user yielding indistinguishable views for this user. A typical situation is illustrated in Figure 2,

However, we do not want to compare a structure of $S y s_{1}$ with arbitrary structures of $S y s_{2}$, but only with certain suitable ones. What suitable means in a concrete situation can be defined by a mapping $f$ from $S y s_{1}$ to $S y s_{2}$. The mapping $f$ is called valid if it maps structures with the same service ports, so that the same user can connect.

Definition 10. (Valid Mappings) A valid mapping between two systems Sys ${ }_{1}$ and Sys $_{2}$ is a function $f:$ Sys $_{1} \rightarrow$ Sys $_{2}$ with $\left(\hat{M}_{2}, S_{2}\right)=f\left(\left(\hat{M}_{1}, S_{1}\right)\right) \Rightarrow S_{1}=S_{2}$. We call $f\left(\left(\hat{M}_{1}, S_{1}\right)\right)$ the corresponding structure of $\left(\hat{M}_{1}, S_{1}\right)$. If the systems are parametrized, we also require $f\left(S y s_{1, k}\right) \subseteq S y s_{2, k}$ for all $k \in \mathbb{N}$.

A technical problem for reactive simulatability is that a correct user of a structure from Sys $_{1}$ might have forbidden ports in the corresponding structure. Configurations where this does not happen are called suitable; we restrict the simulatability definition to those. We omit a rigorous definition for brevity. For a valid mapping $f: S y s_{1} \rightarrow S y s_{2}$, let Conf $^{f}\left(\right.$ Sys $\left._{1}\right)$ be the set of suitable configurations.

We present the definition of indistinguishability for two families of random variables with a common partitioned index set and with versions for concrete security, following [347.12].

Definition 11. (Indistinguishability) Let two families $\left(\operatorname{var}_{x}\right)_{x \in X}$ and $\left(\operatorname{var}_{x}^{\prime}\right)_{x \in X}$ of discrete probability distributions (random variables)

- They are called perfectly indistinguishable iff $\operatorname{var}_{x}=\operatorname{var}_{x}^{\prime}$ for all $x \in X$.

- They are called statistically $\delta$-indistinguishable for a function $\delta: \mathbb{N} \rightarrow \mathbb{R}_{\geq 0}$ iff the statistical distance $\Delta_{\text {stat }}\left(\operatorname{var}_{x}, \operatorname{var}_{x}^{\prime}\right):=\frac{1}{2} \sum_{d \in D_{x}}\left|\operatorname{Pr}\left(\operatorname{var}_{x}=d\right)-\operatorname{Pr}\left(\operatorname{var}_{x}^{\prime}=d\right)\right|$ is at most $\delta(k)$ for all $k$ and all $x \in X_{k}$.

- An algorithm Dis is called a $(t, \delta)$-distinguisher for $\operatorname{var}_{x}$ and $\operatorname{var}_{x}^{\prime}$ for $t \in \mathbb{N}, \delta \in$ $\mathbb{R}_{\geq 0}$, and $x \in X_{k}$ iff its complexity is at most $t$ and

$$
\delta_{x}^{\text {Dis }}:=\left|\operatorname{Pr}\left(\operatorname{Dis}\left(1^{k}, \operatorname{var}_{x}\right)=1\right)-\operatorname{Pr}\left(\operatorname{Dis}\left(1^{k}, \operatorname{var}_{x}^{\prime}\right)=1\right)\right| \geq \delta .
$$


- The distributions are called polynomially indistinguishable iff for all polynomials $t$ and all distinguishers $\left(\operatorname{Dis}_{x}\right)_{x \in X}$ with complexity $t$ in their first parameter, there exists a negligible function $\delta$ such that $\delta_{x}^{\text {Dis }} \leq \delta(k)$ for all $k$ and all $x \in X_{k}$.

We write " $\approx_{y}$ " for indistinguishability with $y=$ perf, $\delta$, or poly, respectively. We write " $\approx$ " if we want to treat all cases together, and we often write "=" for " $\approx$ perf $"$.

We later need that indistinguishability of families of random variables implies indistinguishability of functions of them, e.g., of "parts" of the random variables.

Lemma 1. (Indistinguishability of Derived Distributions) Let var, var' be families of probability distributions with partitioned index set $X$ and a common family of domains $D$, and let $\phi=\left(\phi_{x}\right)_{x \in X}$ be a family of functions $\phi_{x}$ on $D_{x}$ (to strings, but encoding domains as strings is not made explicit). Then the following holds:

- var $\approx_{y} \operatorname{var}^{\prime} \Rightarrow \phi($ var $) \approx_{y} \phi\left(\operatorname{var}^{\prime}\right)$ if $y$ is perf, or a function $\delta$.

- Every $(t, \delta)$-distinguisher $\operatorname{Dis}_{\phi}$ for $\phi\left(\operatorname{var}_{x}\right)$ and $\phi\left(\operatorname{var}_{x}^{\prime}\right)$ gives rise to a $\left(t^{\prime}, \delta\right)$ distinguisher Dis for var $_{\mathrm{x}}$ and var $_{x}^{\prime}$ with $t^{\prime}=t+t_{\phi}(b(k))$, where $t_{\phi}: \mathbb{N} \rightarrow \mathbb{N}$ denotes the complexity of $\phi$, and $b: \mathbb{N} \rightarrow \mathbb{N}$ bounds the length of the random variables, i.e., $|v| \leq b(k)$ for all $v \in D_{x}$ and $x \in X_{k}$.

- var $\approx_{\text {poly }} \operatorname{var}^{\prime} \Rightarrow \phi(\operatorname{var}) \approx_{\text {poly }} \phi\left(\operatorname{var}^{\prime}\right)$ if the random variables are of polynomial length, and $\phi$ is of polynomial complexity.

This is clear for the perfect case, and can be easily shown by computations on statistical distances for the statistical case. For concrete complexity and the computational case, the distinguisher family Dis for the original distributions is defined by $\operatorname{Dis}_{x}\left(1^{k}, v\right):=$ $\operatorname{Dis}_{\phi, x}\left(1^{k}, \phi(v)\right)$ for all $k$ and $x \in X_{k}$, and for $v$ of length at most $b(k)$.

We are now ready to define reactive simulatability for parametrized systems. We require that there exists an extension $f_{C}$ of the valid structure mapping $f$ to a configuration mapping that leaves the user unchanged, i.e., we skolemize the existence of corresponding adversaries in Figure 2. We then consider the families of user views view $\operatorname{conf}_{1}(\mathrm{H})$ and view $_{f_{\mathrm{C}}\left(\operatorname{conf}_{1}\right)}(\mathrm{H})$ where all machines have initial input $1^{k}$ for the security parameter $k$ to which this configuration belongs. Each of these two families contains one well-defined probability distribution for each configuration $\operatorname{conf}_{1}$. Overall these are two families of distributions with the partitioned index set $\operatorname{Conf}^{f}\left(\operatorname{Sys}_{1}\right)=\bigcup_{k \in \mathbb{N}} \operatorname{Conf}^{f}\left(\right.$ Sys $\left._{1, k}\right)$. Similarly, we obtain two families view conf $_{1}, l(\mathbf{H})$ and view $f_{\mathrm{c}}\left(\operatorname{conf}_{1}\right), l(\mathbf{H})$ for $l$-step prefixes of user views.

Definition 12. (Reactive Simulatability) Let parametrized systems $\mathrm{Sys}_{1}$ and $\mathrm{Sys}_{2}$ with a valid mapping $f$ be given. For reactive simulatability, we require that there exists a function $f_{\mathrm{C}}: \operatorname{Conf}^{f}\left(\operatorname{Sys}_{1}\right) \rightarrow \operatorname{Conf}\left(\right.$ Sys $\left._{2}\right)$ with $f_{\mathrm{C}}\left(\right.$ conf $\left._{1}\right)$. struc $=f\left(\right.$ conf $_{1}$. struc $)$ and $f_{\mathrm{C}}\left(\operatorname{conf}_{1}\right) \cdot \mathrm{H}=\operatorname{conf}_{1} \cdot \mathrm{H}$ for all conf $f_{1} \in \operatorname{Conf}^{f}\left(S_{y} s_{1}\right)$, and with the following properties. We say that $f_{\mathrm{C}}$ is a $\tau$-mapping for a structure struc $c_{1}$ and a function $\tau: \mathbb{N} \rightarrow \mathbb{N}$ if the complexity $t_{f_{\mathrm{C}}\left(\operatorname{conf}_{1}\right) \text {.A }}$ is bounded by $\tau\left(t_{\text {conf }_{1} . \mathrm{A}}\right)$ for all conf $f_{1} \in \operatorname{Conf}\left(\operatorname{struc}_{1}\right)$. The entire $f_{C}$ is a $\tau$-mapping for a function $\tau: \mathbb{N}^{2} \rightarrow \mathbb{N}$ iffor all conf $f_{1} \in \operatorname{Conf}^{f}\left(\right.$ Sys $\left._{1}\right)$ we have $t_{f_{\mathrm{C}}\left(\text { conf }_{1}\right) . \mathrm{A}} \leq \tau\left(k, t_{\text {conf }_{1}}\right)$.

We say that $S y s_{1} \geq_{\text {sec }}^{f, y} S y s_{2}$, spoken " $y^{\prime}$-at least as secure as", under the following conditions for different cases of $y$ and $y^{\prime}$, where we abbreviate $\mathrm{H}:=$ conf $_{1} . \mathrm{H}$ : 
a) $y=$ perf and $y^{\prime}=$ "perfectly" iff view conf $_{1}(\mathrm{H})$ and view $_{f_{\mathrm{C}}\left(\text { conf }_{1}\right)}(\mathrm{H})$ are perfectly indistinguishable for every conf $f_{1} \in \operatorname{Conf}^{f}\left(\right.$ Sys $\left._{1}\right)$.

b) $y=\delta$ and $y^{\prime}=$ " $\delta$-statistically" for a function $\delta: \mathbb{N}^{2} \rightarrow \mathbb{R}_{\geq 0}$ iff for every conf $f_{1} \in$ Conf $^{f}\left(\right.$ Sys $\left._{1, k}\right)$ and every $l \in \mathbb{N}$ we have view conf $_{1, l}(\mathrm{H}) \approx_{\delta(k, l)}$ view $_{f_{\mathrm{C}}\left(\text { conf }_{1}\right), l}(\mathrm{H})$.

c) Concrete security: An algorithm $\mathrm{Dis}$ is called a $(t, \delta)$-distinguisher for conf $f_{1} \in$ Conf $^{f}\left(\right.$ Sys $\left._{1, k}\right)$ and $f_{\mathrm{C}}\left(\right.$ conf $\left._{1}\right)$ where $t \in \mathbb{N}$ and $\delta \in \mathbb{R}_{\geq 0}$ iff its complexity is at most $t$ and $\delta_{\text {conf }_{1}}^{\text {Dis }} \geq \delta$ where

$\delta_{\text {conf }}^{\text {Dis }}:=\mid \operatorname{Pr}\left(\operatorname{Dis}\left(1^{k}\right.\right.$, view $\left.\left._{\text {conf }_{1}}(\mathrm{H})\right)=1\right)-\operatorname{Pr}\left(\operatorname{Dis}\left(1^{k}\right.\right.$, view $\left.\left._{f_{\mathrm{c}}\left(\text { conf }_{1}\right)}(\mathrm{H})\right)=1\right) \mid$.

e) $y=$ poly and $y^{\prime}=$ "polynomially" iff for all users $\mathrm{H}$ and adversary $\mathrm{A}$ of polynomial complexity, the views $\left(\text { view }_{\left(\hat{M}, S, \mathrm{H}_{(\hat{M}, S)}, \mathrm{A}_{(\hat{M}, S)}\right)}\left(\mathrm{H}_{(\hat{M}, S)}\right)\right)_{(\hat{M}, S) \in S y s_{1}}$ and view $\left._{f_{\mathrm{C}}\left(\left(\hat{M}, S, \mathrm{H}_{(\hat{M}, S)}, \mathrm{A}_{(\hat{M}, S)}\right)\right)}\left(\mathrm{H}_{(\hat{M}, S)}\right)\right)_{(\hat{M}, S) \in S y s_{1}}$ are polynomially indistinguishable and $f_{\mathrm{C}}$ is a $P$-mapping for a polynomial $P$.

Universal simulatability means that $f_{\mathrm{C}}\left(\right.$ conf $\left._{1}\right)$. A (i.e., $\mathrm{A}_{2}$ in Figure 2) for conf $\mathrm{f}_{1}=$ $\left(\hat{M}_{1}, S, \mathrm{H}, \mathrm{A}_{1}\right)$ only depends on $\hat{M}_{1}, S$, and $\mathrm{A}_{1}$. We write $\geq_{\mathrm{sec}}^{\mathrm{uni}, f, y}$ instead of $\geq_{\mathrm{sec}}^{f, y}$ if we want to emphasize this case.

Where the difference between the types of security is irrelevant, we only write $\geq_{\text {sec }}^{f}$, and we omit the indices $f$ and sec if they are clear from the context.

An essential ingredient in the composition theorem and other uses of the model is a notion of combining several machines into one, and a lemma that this makes no essential difference in views. The combination is defined in a canonical way by considering a combined state space and letting each transition function operate on its respective part. We omit details for brevity. The combination of a set $\hat{M}$ of machines is written $\operatorname{comb}(\hat{M})$ and we sometimes write $\operatorname{comb}\left(\mathrm{M}_{1}, \ldots, \mathrm{M}_{j}\right)$ for $\operatorname{comb}\left(\left\{\mathrm{M}_{1}, \ldots, \mathrm{M}_{j}\right\}\right)$.

Lemma 2. (Machine Combination) Let $\hat{C}$ be a collection without buffers, and $\hat{D} \subseteq \hat{C}$. The view of every set of original machines in $(\hat{C} \backslash \hat{D}) \cup\{\operatorname{comb}(\hat{D})\}$ is the same as in $\hat{C}$. This includes the view of the submachines in $\operatorname{comb}(\hat{D})$, which is well-defined given $\hat{C}$ and $\hat{D}$. The Turing complexity of $\operatorname{comb}(\hat{D})$ is the sum of the complexities of the machines in $\operatorname{comb}(\hat{D})$.

We can now add the notion of blackbox simulatability to Definition 12 . Here $A_{2}$ is given as the combination of a fixed "simulator" Sim and a machine $A_{1}^{\prime}$ that is identical to $A_{1}$ up to port renaming.

Definition 13. (Blackbox Simulatability) With the notation of Definition 12 blackbox simulatability means that we have functions $f_{\mathrm{Sim}}$ from Sys $s_{1}$ to machines (the simulators for the structures) and $f_{\sigma}$ from Sys ${ }_{1}$ to port renaming functions such that for all $\operatorname{conf}_{1}=\left(\hat{M}_{1}, S, \mathrm{H}, \mathrm{A}_{1}\right) \in \operatorname{Conf}^{f}\left(\right.$ Sys $\left._{1}\right)$ we have $f_{\mathrm{C}}\left(\right.$ conf $\left._{1}\right)=\left(\hat{M}_{2}, S, \mathrm{H}, \mathrm{A}_{2}\right)$ with $\left(\hat{M}_{2}, S\right)=f\left(\left(\hat{M}_{1}, S\right)\right)$ and $\mathrm{A}_{2}=\operatorname{comb}\left(\operatorname{Sim}, \mathrm{A}_{1}^{\prime}\right)$ with $\operatorname{Sim}:=f_{\operatorname{Sim}}\left(\left(\hat{M}_{1}, S_{1}\right)\right)$ and $\mathrm{A}_{1}^{\prime}:=f_{\sigma}\left(\left(\hat{M}_{1}, S_{1}\right)\right)\left(\mathrm{A}_{1}\right)$. For computational security, we require that $\mathrm{Sim}$ is polynomialtime, i.e., that the parametrized complexity of $\left(f_{\operatorname{Sim}}\left(\left(\hat{M}_{1}, S\right)\right)\right)_{\left(\hat{M}_{1}, S\right) \in S y s_{1}}$ is polynomially bounded. We write $\geq_{\mathrm{sec}}^{\mathrm{bb}}$ instead of $\geq_{\mathrm{sec}}$ if we want to emphasize this case (with the respective superscripts). 


\subsection{Composition}

When composing several systems, one typically does not want to compose every structure of one system with every structure of the others, but only with certain matching ones. For instance, if the individual machines of $\mathrm{Sys}_{2}$ are implemented on the same physical devices as those of $S y s_{1}$, as usual in a layered distributed system, we only compose structures corresponding to the same set of corrupted physical devices. However, this is not the only conceivable situation. Hence we do not define a composition operator that produces one specific composition, but a set of possible compositions.

Definition 14. (Composability and Composition of Structures) We call structures $\left(\hat{M}_{1}, S_{1}\right), \ldots,\left(\hat{M}_{n}, S_{n}\right)$ composable if $\operatorname{ports}\left(\hat{M}_{i}\right) \cap \operatorname{forb}\left(\hat{M}_{j}, S_{j}\right)=\emptyset$ and $S_{i} \cap$ free $\left(\left[\hat{M}_{j}\right]\right)=S_{j} \cap$ free $\left(\left[\hat{M}_{i}\right]\right)$ for all $i \neq j$. We then define their composition as $\left(\hat{M}_{1}, S_{1}\right)\|\ldots\|\left(\hat{M}_{n}, S_{n}\right):=(\hat{M}, S)$ with $\hat{M}:=\hat{M}_{1} \cup \ldots \cup \hat{M}_{n}$ and $S:=$ $\left(S_{1} \cup \ldots \cup S_{n}\right) \cap \operatorname{free}([\hat{M}])$.

We now define the composition of variably many systems, i.e., there is a potentially infinite supply of systems from which a finite number $P(k)$ is chosen for composition for each security parameter $k$.

Definition 15. (Parametrized Composition of Systems) Let a sequence Sysseq = $\left(\text { Sys }^{(i)}\right)_{i \in \mathbb{N}}$ be given where each Sys ${ }^{(i)}$ is a parametrized system, and let $P: \mathbb{N} \rightarrow \mathbb{N}$ be a function. Then a P-sized composition of Sysseq is a parametrized system Sys ${ }^{*}$ where for all $k \in \mathbb{N}$, every structure $\left(\hat{M}^{*}, S^{*}\right) \in S y s_{k}^{*}$ has a unique representation $\left(\hat{M}^{*}, S^{*}\right)=\left(\hat{M}_{1}, S_{1}\right)\|\ldots\|\left(\hat{M}_{P(k)}, S_{P(k)}\right)$ with composable structures $\left(\hat{M}_{i}, S_{i}\right) \in \operatorname{Sys}_{k}^{(i)}$ for $i=1, \ldots, P(k)$. We call $\left(\hat{M}_{i}, S_{i}\right)$ the restriction of $\left(\hat{M}^{*}, S^{*}\right)$ to $\operatorname{Sys}^{(i)}$ and write $\left(\hat{M}_{i}, S_{i}\right)=\left(\hat{M}^{*}, S^{*}\right) \Gamma_{\text {Sys }}{ }^{(i)}$.

If the systems $S y s^{(i)}$ have a joint bounding function $Q$, then $P \cdot Q$ is a bounding function for $S y s^{*}$. In particular, if $P$ and $Q$ are polynomials, then $S y s^{*}$ is polynomial-time.

\section{General Composition Theorem for Blackbox Simulatability}

In this section, we show that reactive blackbox simulatability is consistent with the composition of a parametrized number of systems, in particular polynomially many in the computational case. The basic idea is the following: Assume that we have proven that a potentially infinite supply of systems $S y s^{(i)}$ are as secure as systems $S y s^{\prime(i)}$ in the sense of black-box simulatability. Now we want to use $S y s^{(i)}$ as a secure replacement for $S y s^{\prime(i)}$, i.e., as an implementation of the ideal system $S y s^{\prime(i)}$. The following theorem shows that such modular proofs are possible. The situation is shown in the upper part of Figure 3 .

Additional conditions in the theorem are that all corresponding structures are composable and that, for the polynomial case, the security of the system is in certain sense uniform.

\footnotetext{
${ }^{2}$ The first condition makes one structure a valid user of another. The second one excludes cases where $p \in$ free $\left(\left[\hat{M}_{i}\right]\right) \cap$ free $\left(\left[\hat{M}_{j}\right]\right)$ (e.g., a clock port for a connection between these structures) and $p \in S_{i}$ but $p \notin S_{j}$.
} 
Theorem 1. (Secure Parametrized Composition, Blackbox Case) Let Sysseq = $\left(\text { Sys }^{(i)}\right)_{i \in \mathbb{N}}$ and Sysseq ${ }^{\prime}=\left(\text { Sys }^{\prime(i)}\right)_{i \in \mathbb{N}}$ be sequences of parametrized systems. Let $f=\left(f^{(i)}\right)_{i \in \mathbb{N}}$ be a sequence of valid mappings $f^{(i)}:$ Sys ${ }^{(i)} \rightarrow$ Sys ${ }^{(i)}$, and let Sys ${ }^{(i)} \geq_{\text {sec }}^{\text {bb, }}{ }^{(i)}, y_{i} S_{\text {Sys }}{ }^{\prime(i)}$ for all $i \in \mathbb{N}$.

Let $P: \mathbb{N} \rightarrow \mathbb{N}$, and let Sys ${ }^{\#}$ and Sys ${ }^{*}$ denote the P-sized compositions of Sysseq and Sysseq', respectively. Assume that the following structural conditions hold for all $k \in \mathbb{N}$ and every structure $\left(\hat{M}^{\#}, S\right) \in$ Sys $_{k}^{\#}:$ Let its restrictions be $\left(\hat{M}_{i}, S_{i}\right):=$ $\left(\hat{M}^{\#}, S\right) \Gamma_{\text {Sys }}(i)$ and the corresponding structures $\left(\hat{M}_{i}^{\prime}, S_{i}\right):=f^{(i)}\left(\left(\hat{M}_{i}, S_{i}\right)\right)$ for all $i \leq P(k)$. Then the composition

$$
f^{\#}\left(\left(\hat{M}^{\#}, S\right)\right):=\left(\hat{M}_{1}^{\prime}, S_{1}\right)\|\cdots\|\left(\hat{M}_{P(k)}^{\prime}, S_{P(k)}\right)
$$

exists and lies in Sys $k_{k}^{*}$. Furthermore, $\left(\hat{M}_{i}, S_{i}\right)$ and $\left(\hat{M}_{j}^{\prime}, S_{j}\right)$ must be composable for $j \neq i$, and $\operatorname{ports}\left(\hat{M}_{i}^{\prime}\right) \cap S_{j}^{c}=\operatorname{ports}\left(\hat{M}_{i}\right) \cap S_{j}^{c}$ for all $j \neq i$. Then we have

$$
\text { Sys }^{\#} \geq_{\mathrm{sec}}^{\mathrm{bb}, f^{\#}, y} \text { Sys }^{*}
$$

a) with $y=$ perf if $y_{i}=$ perf for all $i \in \mathbb{N}$.

b) with $y=P(k) \cdot \delta(k, b(k))$ if all $y_{i}$ are bounded by a function $\delta: \mathbb{N}^{2} \rightarrow \mathbb{R}_{\geq 0}$, and where $b(k)$ is the sum of the complexity of the systems, the user, and the simulators.

c) With concrete complexity: For every conf $f^{\#} \in \operatorname{Conf}^{f^{\#}}\left(S y s_{k}^{\#}\right), a(t, \delta)$-distinguisher for conf $\#$ and $f_{\mathrm{C}}\left(\right.$ conf $\left.f^{\#}\right)$ gives rise to a $\left(t^{\prime}, \delta^{\prime}\right)$-distinguisher for conf ${ }^{(i)}$ and $f_{\mathrm{C}}\left(\operatorname{conf}^{(i)}\right)$ for a conf ${ }^{(i)} \in \operatorname{Conf}\left(\right.$ Sys $\left._{k}^{(i)}\right)$ with $\delta^{\prime}=\frac{\delta}{P(k)}$ and $t^{\prime}=t+b^{\prime}(k)$, where $b^{\prime}(k)$ is a polynomial independent of $t_{\text {conf \#.A. (Details are given in the proof.) }}$

d) with $y=$ poly if $y_{i}=$ poly for all $i \in \mathbb{N}$ and under the following conditions: The function $P$ is polynomially bounded, and the systems $S y s^{(i)}$ have a joint bounding polynomial $Q$. The complexities of the simulator families induced by the mappings $f_{\text {Sim }}^{(i)}$ are bounded by a joint polynomial $Q_{\text {Sim. The distinguishing probabilities of }}$ the system pairs $\left(S y s^{(i)}, S^{\prime} s^{(i)}\right)$ are uniformly bounded, i.e., for all polynomials $t$ there exists a negligible function $\delta$ such that for all distinguishers Dis, all $i, k \in \mathbb{N}$, and all conf $=\left(\hat{M}_{i}, S_{i}, \mathrm{H}, \mathrm{A}\right) \in \operatorname{Conf}^{f^{(i)}}\left(\right.$ Sys $\left._{k}^{(i)}\right)$ we have $\left(t_{\text {Dis }} \leq t(k) \wedge t_{\mathrm{H}} \leq\right.$ $\left.t(k) \wedge t_{\mathrm{A}} \leq t(k)\right) \Rightarrow \delta_{\text {conf }}^{\text {Dis }} \leq \delta(k)$ (recall Definition [2] $)$.

The first statement to be proved is extracted into the following lemma.

Lemma 3. Under the conditions of Theorem $\square$ the mapping $f^{\#}$ is a valid mapping between Sys ${ }^{\#}$ and Sys*.

The proof is straightforward as in [30], but heavy on notation. Hence we omit it in this short version. Recall that blackbox simulatability was defined by a function that selects one fixed simulator for each structure (Definition 13).

Definition 16. (Simulator and Corresponding Configurations) Under the conditions of Theorem $\square$ and for all $i \in \mathbb{N}$, let $f_{\mathrm{Sim}}^{(i)}$ and $f_{\mathrm{A}}^{(i)}$ be the simulator and renaming functions from which $f_{\mathrm{C}}^{(i)}$ is composed by blackbox simulatability. We compose them 
conf \#

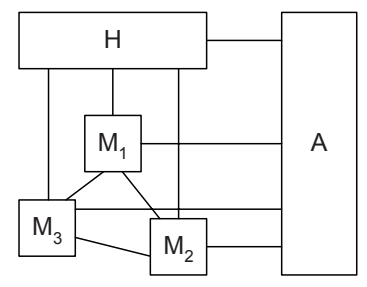

$\operatorname{conf}_{i}^{\text {hyb }}$

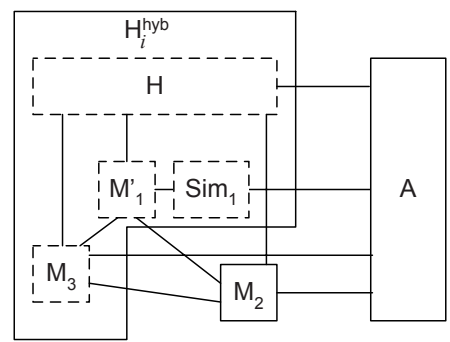

conf $^{*}$

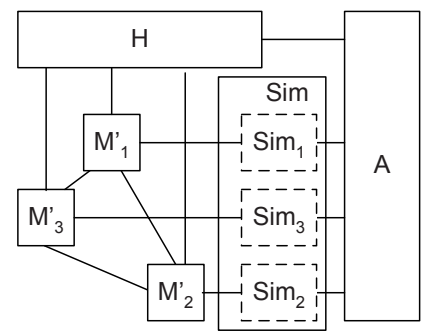

$\operatorname{conf}_{i}^{\prime \text { hyb }}$

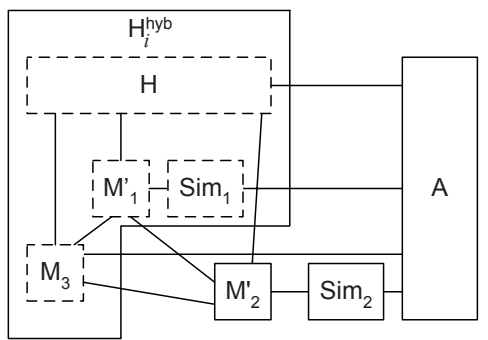

Fig. 3. Configurations in the composition theorem for blackbox simulatability.

into functions $f_{\mathrm{Sim}}^{\#}$ and $f_{\mathrm{A}}^{\#}$ on Sys ${ }^{\#}$ as follows: Given $k \in \mathbb{N}$ and $\left(\hat{M}^{\#}, S\right) \in S y s_{k}^{\#}$, let $\operatorname{Sim}_{i}:=f_{\operatorname{Sim}}^{(i)}\left(\left(\hat{M}_{i}, S_{i}\right)\right)$ for all $i \leq P(k)$, and let

$$
f_{\text {Sim }}^{\#}\left(\left(\hat{M}^{\#}, S\right)\right):=\operatorname{comb}\left(\operatorname{Sim}_{1}, \ldots, \operatorname{Sim}_{P(k)}\right) ;
$$

further let $f_{\mathrm{A}}^{\#}:=f_{\mathrm{A}}^{(P(k))} \circ \cdots \circ f_{\mathrm{A}}^{(1)}$. Let $f_{\mathrm{C}}^{\#}$ be constructed from $f^{\#}, f_{\mathrm{Sim}}^{\#}$, and $f_{\mathrm{A}}^{\#}$ by the equations in Definition 13 (blackbox simulatability).

The complexity $t_{\mathrm{Sim}}$ of the simulator is $t_{\mathrm{Sim}}(k)=\sum_{i=1}^{P(k)} t_{\operatorname{Sim}_{i}}(k)$ by Lemma 2. In the polynomial case, there exists a polynomial $Q_{\mathrm{Sim}}$ such that $t_{\operatorname{Sim}_{i}} \leq Q_{\mathrm{Sim}}$ for all $i$, hence $t_{\text {Sim }}(k)$ is polynomially bounded by $P(k) \cdot Q_{\operatorname{Sim}}(k)$.

We also omit the technical proof that indeed $f_{C}^{\#}: \operatorname{Conf}^{f^{\#}}\left(\right.$ Sys $\left.^{\#}\right) \rightarrow \operatorname{Conf}\left(\right.$ Sys $\left.^{*}\right)$ in Definition 16. It is nevertheless interesting that these proof parts that verify the compatibility of channels and the difference of service ports and adversary ports in compositions make up the major part of a rigorous proof, while the cryptographic aspects are shorter and more standard.

Now we can concentrate on proving that the simulator simulates correctly. The proof consists of a hybrid argument as first used in [15], i.e., we construct intermediate configurations that differ only in the machines of one system.

Proof (Theorem प). Let a configuration conf $f^{\#}=\left(\hat{M}^{\#}, S, \mathrm{H}, \mathrm{A}\right) \in \operatorname{Conf}^{f^{\#}}\left(\right.$ Sys $\left._{k}^{\#}\right)$ be given and $\operatorname{conf}^{*}:=f_{\mathrm{C}}^{\#}\left(\right.$ conf $\left.^{\#}\right)$ the corresponding configuration according to Definition 16. Let the sub-structures $\left(\hat{M}_{i}, S_{i}\right)$ and $\left(\hat{M}_{i}^{\prime}, S_{i}^{\prime}\right)$, the simulators $\operatorname{Sim}_{i}$, and functions $f_{z}^{x}$ with various indices be defined as in the formulation of the theorem and Definition 16 
Furthermore, let $\left(\hat{M}^{*}, S\right):=f^{*}\left(\left(\hat{M}^{\#}, S\right)\right)$ and $\operatorname{Sim}:=f_{\text {Sim }}^{\#}\left(\left(\hat{M}^{\#}, S\right)\right)$. Then we have conf $f^{*}=\left(\hat{M}^{*}, S, \mathrm{H}, \operatorname{comb}\left(\operatorname{Sim}, f_{\mathrm{A}}^{\#}(\mathrm{~A})\right)\right)$; recall that $f_{\mathrm{A}}^{\#}$ is just a port renaming; hence Figure 3 simplifies it to $A$.

The outline of the hybrid argument is as follows.

1. We define hybrid configurations conf $f_{i}^{\text {hyb }}$ of $S y s^{(i)}$ and $\operatorname{conf}_{i}^{\text {hyb }}$ of $S y s^{(i)}$ for $i=$ $1, \ldots, P(k)$. In $c o n f_{i}^{\text {hyb }}$ the first $i-1$ real structures have already been replaced with their ideal counterparts, while in $\operatorname{conf}_{i}^{\text {hyb }}$ also the $i$-th structure has been replaced. To make these configurations correct configurations of the respective systems, all other machines are grouped into an overall hybrid user $\mathrm{H}_{i}^{\text {hyb }}$ as shown at the bottom of Figure 3 for $i=2$ and $P(k)=3$.

2. We show that these are correct and corresponding configurations with respect to the given blackbox simulatability between $\mathrm{Sys}^{(i)}$ and $S y s^{(i)}$.

3. We show that the views of $\mathrm{H}$ in $\operatorname{conf}_{i}^{\text {hyb }}$ and $\operatorname{conf}_{i+1}^{\text {hyb }}$ are equal for $i=1, \ldots, P(k)-$ 1. Moreover, we show that the views of $\mathrm{H}$ are equal in conf $\#$ and conf $f_{1}^{\text {hyb }}$, and in $\operatorname{conf}_{P(k)}^{\text {hyb }}$ and conf ${ }^{*}$. This gives a kind of indistinguishability chain (for one configuration)

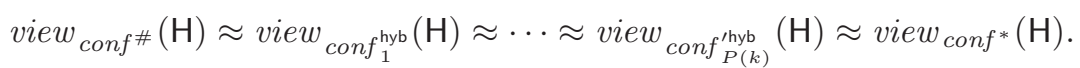

4. We show that this implies indistinguishability between first and last elements.

We now explain these steps in more detail.

Step 1: For $i=1, \ldots, P(k)$, let the machine collection for the $i$-th hybrid user be $\hat{H}_{i}:=$ $\{\mathrm{H}\} \cup \bigcup_{1 \leq j<i} \hat{M}_{j}^{\prime} \cup\left\{\operatorname{Sim}_{j} \mid 1 \leq j<i\right\} \cup \bigcup_{i<j \leq P(k)} \hat{M}_{j}$, and let $\mathrm{H}_{i}^{\text {hyb }}:=\operatorname{comb}\left(\hat{H}_{i}\right)$. Furthermore let $\mathrm{A}_{i}:=f_{\mathrm{A}}^{(i-1)} \circ \cdots \circ f_{\mathrm{A}}^{(1)}(\mathrm{A})$ and $\mathrm{A}_{i}^{\prime}:=f_{\mathrm{A}}^{(i)}\left(\mathrm{A}_{i}\right)$. Then we define the hybrid configurations as

$$
\begin{aligned}
\operatorname{conf}_{i}^{\text {hyb }} & :=\left(\hat{M}_{i}, S_{i}, \mathrm{H}_{i}^{\text {hyb }}, \mathrm{A}_{i}\right) \\
\operatorname{conf}_{i}^{\text {hyb }} & :=\left(\hat{M}_{i}^{\prime}, S_{i}, \mathrm{H}_{i}^{\text {hyb }}, \operatorname{comb}\left(\operatorname{Sim}_{i}, \mathrm{~A}_{i}^{\prime}\right)\right) .
\end{aligned}
$$

For the computational case, we have to show that the family of $\mathrm{H}_{i}^{\text {hyb }}$ is polynomial-time. This holds since $t_{\mathrm{H}_{i}^{\text {hyb }}} \leq t_{\mathrm{H}}+t_{\text {Sim }}+t_{\hat{M}^{\#}}+t_{\hat{M}^{*}}$ by Lemma 2, where each addend is polynomially bounded by assumption.

Step 2: We have to show that $\operatorname{conf}_{i}^{\text {hyb }} \in \operatorname{Conf}^{f_{i}}\left(\operatorname{Sys}_{i}\right)$ and $\operatorname{conf}_{i}^{\text {'hyb }} \in \operatorname{Conf}\left(S y s_{i}^{\prime}\right)$, i.e., essentially that the hybrid users do not use non-service ports. In this short version, we omit this proof. Then the definition of $\operatorname{conf}_{i}^{\text {hyb }}$ and $\operatorname{conf}_{i}^{\text {hyb }}$ immediately implies

$$
\operatorname{conf}_{i}^{\text {hyb }}=f_{\mathrm{C}}^{(i)}\left(\operatorname{conf}_{i}^{\text {hyb }}\right),
$$

i.e., these are indistinguishable configurations under the given blackbox simulatability between Sys $^{(i)}$ and $\operatorname{Sys}^{\prime(i)}$. 
Step 3: The configurations conf $f_{i}^{\text {hyb }}$ and conf $f_{i+1}^{\text {hyb }}$ consist of the same collection of machines $\hat{C}_{i}:=\hat{H}_{i} \cup\left\{\hat{M}_{i}^{\prime}, \operatorname{Sim}_{i}, \mathrm{~A}_{i}^{\prime}\right\}$. Combining them in different ways does not alter the view of $\mathrm{H}$ by Lemma 2 Thus we have

$$
\text { view }_{\text {conf }}^{\text {hyyb }}(\mathbf{H})=\text { view }_{\text {conf hyb }}^{\text {hyb }}(\mathbf{H})
$$

for all $i \in\{1, \ldots, P(k)\}$, and similarly

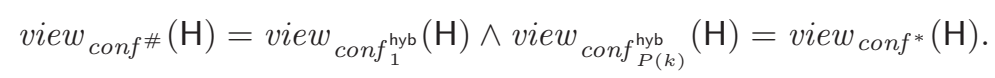

Step 4: We now distinguish the type of the given simulatability relations $S y s^{(i)} \geq_{\mathrm{sec}}^{\mathrm{bb}, f^{(i)}, y_{i}}$ Sys $^{\prime(i)}$.

For perfect simulatability, Equation (1) gives us view $\operatorname{conf}_{i}^{\text {hyb }}(\mathrm{H})=\operatorname{view}_{\text {conf }}{ }_{i}^{\text {hyb }}(\mathrm{H})$ for all $i$. With Equations (2) and (3) this yields view $w_{\text {conf } \#(}(\mathbf{H})=v_{\text {view }}$ conf* $^{*}(\mathbf{H})$. This result for an arbitrary fixed configuration conf ${ }^{\#}$ implies equality of all families of such views.

For statistical simulatability, let $S y s^{(i)}$ be $\delta_{i}$-statistically at least as secure as $S y s^{(i)}$. Let $l \in \mathbb{N}$. For prefixes of length $l$ and $v$ ranging over the potential views of this length, we abbreviate $q_{v}^{\#}:=\operatorname{Pr}\left(\right.$ view $\left._{\text {conf }^{\#}, l}(\mathrm{H})=v\right)$, and $q_{v}^{*}:=\operatorname{Pr}\left(\right.$ view $\left._{\text {conf }^{*}, l}(\mathrm{H})=v\right)$, and $q_{i, v}:=\operatorname{Pr}\left(\right.$ view $\left._{\text {conf }_{i}^{\text {hyb }}, l}(\mathrm{H})=v\right)$ and $q_{i, v}^{\prime}:=\operatorname{Pr}\left(v_{i e} w_{\text {conf }_{i}^{\text {hyb }}, l}(\mathrm{H})=v\right)$ for all $i$. For all potential views $v$, we have $q_{i, v}^{\prime}=q_{i+1, v}$ and $q_{v}^{\#}=q_{1, v}$ and $q_{P(k), v}^{\prime}=q_{v}^{*}$ by Equations (2) and (3). The desired statistical distance is

$$
\begin{aligned}
\delta_{\text {stat }}\left(\text { conf }^{\#}\right) & :=\frac{1}{2} \sum_{v}\left|q_{v}^{\#}-q_{v}^{*}\right| \\
= & \frac{1}{2} \sum_{v}\left|q_{1, v}-q_{2, v}+q_{2, v}-q_{3, v}+\cdots+q_{P(k), v}-q_{P(k), v}^{\prime}\right| \\
\leq & \frac{1}{2} \sum_{v}\left(\left|q_{1, v}-q_{2, v}\right|+\left|q_{2, v}-q_{3, v}\right|+\cdots+\left|q_{P(k), v}-q_{P(k), v}^{\prime}\right|\right) \\
& =\sum_{i=1}^{P(k)} \frac{1}{2} \sum_{v}\left|q_{i, v}-q_{i, v}^{\prime}\right| \\
& =\sum_{i=1}^{P(k)} \Delta_{\text {stat }}\left(\text { view }_{\text {conf }_{i}^{\text {hyb }}, l}(\mathrm{H}), \text { view }_{\text {conf }_{i}^{\text {'hyb }}, l}(\mathrm{H})\right) .
\end{aligned}
$$

With Lemma 1 this gives

$$
\delta_{\text {stat }}\left(\text { conf }^{\#}\right) \leq \sum_{i=1}^{P(k)} \Delta_{\text {stat }}\left(\text { view }_{\text {conf }_{i}^{\text {hyb }}, l_{i}}\left(\mathrm{H}_{i}^{\text {hyb }}\right), \text { view }_{\text {conf }_{i}^{\text {hyb }}, l_{i}}\left(\mathrm{H}_{i}^{\text {hyb }}\right)\right) \leq \sum_{i=1}^{P(k)} \delta\left(k, l_{i}\right) \text {, }
$$

where the $l_{i}$ are sufficiently large numbers to ensure that the $l$-step prefix of the view of $\mathrm{H}$ in $\operatorname{conf} f_{i}^{\text {hyb }}$ is a subsequence of the $l_{i}$-step prefix of the view of $\mathrm{H}_{i}^{\text {hyb }}$. A general 
bound is the complexity of $\mathrm{H}_{i}^{\text {hyb }}$, which is bounded by $b:=t_{\mathrm{H}}+t_{\hat{M} \#}+t_{\hat{M}^{*}}+t_{\mathrm{Sim}}$. This implies $\delta_{\text {stat }}\left(\right.$ conf $\left.{ }^{\#}\right) \leq P(k) \cdot \delta(k, b(k))$ as desired.

For concrete complexity and for a $\left(t, \Delta^{\text {Dis }}\right)$-distinguisher Dis, we have by definition

$$
\Delta^{\text {Dis }} \leq \mid \operatorname{Pr}\left(\operatorname{Dis}\left(1^{k}, \text { view }_{\text {conf }}(\mathrm{H})\right)=1\right)-\operatorname{Pr}\left(\operatorname{Dis}\left(1^{k}, \text { view }_{\text {conf }}(\mathrm{H})\right)=1\right) \mid .
$$

We abbreviate $q^{\#}:=\operatorname{Pr}\left(\operatorname{Dis}\left(1^{k}\right.\right.$, view conf $\left.\left.(\mathrm{H})\right)=1\right)$ and $q^{*}:=\operatorname{Pr}\left(\operatorname{Dis}\left(1^{k}\right.\right.$, view $\left.\left._{\text {conf* }}(\mathrm{H})\right)=1\right)$, and $q_{i}:=\operatorname{Pr}\left(\operatorname{Dis}\left(1^{k}\right.\right.$, view conf $\left.\left._{i}^{\text {hyb }}(\mathrm{H})\right)=1\right)$ and $q_{i}^{\prime}:=\operatorname{Pr}\left(\operatorname{Dis}\left(1^{k}\right.\right.$, view $\left._{\text {conf }}^{\text {'hyb }}(\mathrm{H})\right)=1$ ) for all $i$, and $\Delta_{i}:=\left|q_{i}-q_{i}^{\prime}\right|$. Now Equations (2) and (3) yield

$$
\begin{aligned}
\Delta^{\text {Dis }} & =\left|q^{\#}-q^{*}\right|=\left|q_{1}-q_{2}+q_{2}-q_{3}+q_{3}+\cdots+q_{P(k)}-q_{P(k)}^{\prime}\right| \\
& \leq\left|q_{1}-q_{2}\right|+\left|q_{2}-q_{3}\right|+\cdots+\left|q_{P(k)}-q_{P(k)}^{\prime}\right|=\Delta_{1}+\Delta_{2}+\cdots+\Delta_{P(k)} .
\end{aligned}
$$

This implies that there exists some $i$ with $\Delta_{i} \geq \frac{\Delta^{\text {Dis }}}{P(k)}$.

We can now consider Dis as a $\left(t, \Delta_{i}\right)$-distinguisher $\operatorname{Dis}_{\phi}^{(i)}$ of a function $\phi$ of views of the actual user $\mathrm{H}_{i}^{\text {hyb }}$ of the $i$-th hybrid systems. Here $\phi$ is defined by $\phi(v):=v\left\lceil_{\mathrm{H}}\right.$, i.e., the restriction to the view of $\mathrm{H}$. The complexity $t_{\phi}$ of $\phi$ is linear. Hence Lemma 1 implies that there exists a $\left(t_{i}, \Delta_{i}\right)$-distinguisher Dis ${ }^{(i)}$ for view ${\text { conf } f_{i}^{\text {hyb }}}\left(\mathrm{H}_{i}^{\text {hyb }}\right)$ and view conf $_{i}^{\text {hyb }}\left(\mathrm{H}_{i}^{\text {hyb }}\right)$ with $t_{i}=t+b^{\prime}(k)$, where $b^{\prime}(k)$ bounds the length of the views of $\mathrm{H}_{i}^{\text {hyb }}$. The complexity $t_{\mathrm{H}_{i}^{\text {hyb }}}$ of $\mathrm{H}_{i}^{\text {hyb }}$ is bounded by $b=t_{\mathrm{H}}+t_{\hat{M} \#}+t_{\hat{M}^{*}}+t_{\text {Sim }}$, and above we showed $t_{\text {Sim }} \leq$ $P \cdot Q_{\text {sim }}$. The length of runs and thus views in our current representation is bounded by the square of this complexity (but this might be improvable by tighter encoding). This yields the desired polynomial bound $b^{\prime}(k)$ independent of the adversary complexity.

For polynomial simulatability, let $\mathrm{H}$, A be a user and an adversary for Sys ${ }^{\#}$ of complexity $t_{\mathrm{H}}$ and $t_{\mathrm{A}}$, and let $t$ be a polynomial and Dis a distinguisher family of complexity $t$. Then the functions $t_{\mathrm{H}_{i}^{\text {hyb }}}, t_{i}$, and $t_{\mathrm{A}_{i}}=t_{\mathrm{A}}$ are polynomials. By assumption, there exists a negligible function $\delta$ that uniformly bounds the advantage of distinguishers for the given system pairs for the complexity function $\max \left(t_{i}, t_{\mathrm{H}_{i}^{\text {hyb }}}, t_{\mathrm{A}_{i}}\right)$. Now let a configuration conf $\#=\left(\hat{M}^{\#}, S, \mathrm{H}_{\left(\hat{M}^{\#}, S\right)}, \mathrm{A}_{\left(\hat{M}_{\#, S}\right)}\right)$ be given. The concrete security considerations and Equation (1) imply $\Delta_{i}=\delta_{\text {conf }_{i}^{\text {hyb }}}^{\text {ii(i) }^{(i)}} \leq \delta(k)$, and therefore $\delta_{\text {conf }}^{\text {Dis }} \leq$ $P(k) \cdot \delta(k)$ is negligible. This proves the desired polynomial indistinguishability of the families of user views over Sys ${ }^{\#}$ and Sys*.

\section{From Black-Box to Universal Simulatability}

We now show a relation between universal simulatability and black-box simulatability. It allows us to apply our general composition theorem to universal simulatability under reasonable assumptions, but it also is of independent interest. More precisely, we show that universal simulatability for two parametrized systems $S y s_{1}$ and $S y s_{2}$ is equivalent to black-box simulatability if $S y s_{1}$ fulfills the following structural requirements: Whenever a clock-out port of a structure $\left(\hat{M}_{1}, S_{1}\right) \in S y s_{1}$ is contained in $\bar{S}_{1}^{c}$, then so is either the corresponding input or output port. This means that the adversary is not allowed to 
schedule messages of a connection where it is neither the sender nor the recipient. This condition is naturally fulfilled for insecure channels, since the adversary is inserted between the connections of two machines of the system.

Theorem 2. (Relating Black-box and Universal Simulatability) Let Sys ${ }_{1}$, Sys $_{2}$ be two parametrized systems with a valid mapping $f$, where for every structure $\left(\hat{M}_{1}, S_{1}\right) \in$ Sys $_{1}$, we have $\mathrm{p}^{\triangleleft} ! \in \bar{S}_{1}^{c} \Rightarrow\left(\mathrm{p} ? \in \bar{S}_{1}^{c} \vee \mathrm{p} ! \in \bar{S}_{1}^{c}\right)$. Then Sys ${ }_{1} \geq_{\mathrm{sec}}^{\mathrm{bb}, f, y}$ Sys $_{2}$ iff Sys $_{1} \geq_{\mathrm{sec}}^{\mathrm{uni}_{1}, y}$ Sys $_{2}$ for $y=$ perf or a function $\delta$ and also for $y=$ poly if $S y s_{1}$ is polynomial-time.

For concrete security, if $\geq_{\mathrm{sec}}^{\mathrm{uni}, f}$ is given with a $\tau$-mapping $f_{\mathrm{C}}$, then we obtain $\geq_{\mathrm{sec}}^{\mathrm{bb}, f}$ with simulator complexity $\tau\left(t_{\text {Sys }_{1}}\right)$, and $a(t, \delta)$-distinguisher for the views in the blackbox case gives rise to a $\left(t^{\prime}, \delta\right)$-distinguisher for the views in the universal case where $t^{\prime}$ is the sum of $t$ and the view length of $\mathrm{H}$ and $\mathrm{A}$.

Proof. The left-to-right direction is clear by definition. The difficult direction is to show that universal simulatability implies black-box simulatability. Due to lack of space, we can only present a short sketch. This direction essentially consists of four steps:

1. Let a configuration $\operatorname{conf}_{1}=\left(\hat{M}_{1}, S, \mathrm{H}, \mathrm{A}_{1}\right)$ of the sub-system $S y s_{1, k}$ be given. We first derive another configuration conf ${ }_{1}^{\text {uni }}=\left(\hat{M}_{1}, S, \mathrm{H}^{\text {uni }}, \mathrm{A}_{1}^{\prime}\right)$ of $S y s_{1}$ as follows: We insert a machine $\mathrm{TS}_{P, b, k}$, called transparent scheduler, into the connections between $A_{1}$ and the simple ports in $\bar{S}_{1}$. It forwards messages between machines of the structure and the adversary. Its parameters $P$ and $b$ correspond to the ports that the transparent scheduler connects to and a bound on its runtime, which is the joint runtime of the machines in $\hat{M}_{1}$. This machine only depends on $\hat{M}_{1}, S$, and $k$. The new user is the combination $\mathrm{H}^{\text {uni }}:=\operatorname{comb}\left(\mathrm{H}, \mathrm{A}_{1}\right)$, and the new adversary is $\mathrm{A}_{1}^{\prime}:=\mathrm{TS}_{P, b, k}$. We show that the views of both $\mathrm{H}$ and $\mathrm{A}_{1}$ are identical in the two configurations.

2. We now show that $\operatorname{conf}_{1}^{\text {uni }} \in \operatorname{Conf}^{f}\left(S y s_{1}\right)$ and apply the precondition $S y s_{1} \geq_{\text {sec }}^{\text {uni, } f}$ $S y s_{2}$. This yields an indistinguishable configuration conf $_{2}^{\text {uni }}$ of $S y s_{2}$ with a new adversary $\mathrm{A}_{2}$. By the definition of universal simulatability, $\mathrm{A}_{2}$ only depends on $\hat{M}_{1}$, $S$ and on $\mathrm{A}_{1}^{\prime}=\mathrm{TS}_{P, b, k}$. Since $\mathrm{TS}_{P, b, k}$ only depends on $\hat{M}_{1}$ and $S$, the adversary $\mathrm{A}_{2}$ also only depends on $\hat{M}_{1}$ and $S$.

3. We obtain a configuration $\operatorname{conf}_{2}$ with the original user and a simulator from conf ${ }_{2}^{\text {uni }}$ by reversing the combination of $\mathrm{H}$ and $\mathrm{A}_{1}$ into $\mathrm{H}^{\text {uni }}$, and by defining the simulator as $\operatorname{Sim}:=A_{2}$. We show that this does not affect the view of $H$.

4. Combining several equalities between views of $\mathrm{H}$ in different configurations and one indistinguishability gives the same class of indistinguishability.

Summarized statements follow from this treatment per configuration, i.e., with concrete security (although details are omitted here), as usual.

Acknowledgments. We thank Anupam Datta, Dennis Hofheinz, Ralf Küsters, John Mitchell, Jörn Müller-Quade, Dusko Pavlovic and Rainer Steinwandt for interesting discussions. 


\section{References}

1. M. Abadi and L. Lamport. Conjoining specifications. ACM Transactions on Programming Languages and Systems, 17(3):507-534, 1995.

2. M. Backes and C. Jacobi. Cryptographically sound and machine-assisted verification of security protocols. In Proc. 20th Annual Symposium on Theoretical Aspects of Computer Science (STACS), volume 2607 of LNCS, pages 675-686. Springer, 2003.

3. M. Backes and B. Pfitzmann. Computational probabilistic non-interference. In Proc. 7th European Symposium on Research in Computer Security (ESORICS), volume 2502 of LNCS, pages 1-23. Springer, 2002.

4. M. Backes and B. Pfitzmann. Intransitive non-interference for cryptographic purposes. In Proc. 24th IEEE Symposium on Security \& Privacy, pages 140-152, 2003.

5. M. Backes, B. Pfitzmann, M. Steiner, and M. Waidner. Polynomial fairness and liveness. In Proc. 15th IEEE Computer Security Foundations Workshop (CSFW), pages 160-174, 2002.

6. D. Beaver. Secure multiparty protocols and zero knowledge proof systems tolerating a faulty minority. Journal of Cryptology, 4(2):75-122, 1991.

7. M. Bellare, J. Killian, and P. Rogaway. The security of cipherblock chaining. In Advances in Cryptology: CRYPTO '94, volume 839 of LNCS, pages 341-358. Springer, 1994.

8. M. Bellare and P. Rogaway. Optimal asymmetric encryption. In Advances in Cryptology: EUROCRYPT '94, volume 950 of LNCS, pages 92-111. Springer, 1994.

9. R. Canetti. Security and composition of multiparty cryptographic protocols. Journal of Cryptology, 3(1):143-202, 2000.

10. R. Canetti. Universally composable security: A new paradigm for cryptographic protocols. In Proc. 42nd IEEE Symposium on Foundations of Computer Science (FOCS), pages 136-145, 2001. Extended version in Cryptology ePrint Archive, Report 2000/67, http://eprint.iacr.org/

11. A. Datta, A. Derek, J. C. Mitchell, and D. Pavlovic. Secure protocol composition (extended abstract). In Proc. 1st ACM Workshop on Formal Methods in Security Engineering (FMSE), pages 11-23, 2003.

12. O. Goldreich. Foundations of Cryptography: Basic Tools. Cambridge University Press, 2001.

13. O. Goldreich, S. Micali, and A. Wigderson. How to play any mental game-or-a completeness theorem for protocols with honest majority. In Proc. 19th Annual ACM Symposium on Theory of Computing (STOC), pages 218-229, 1987.

14. S. Goldwasser and L. Levin. Fair computation of general functions in presence of immoral majority. In Advances in Cryptology: CRYPTO '90, volume 537 of LNCS, pages 77-93. Springer, 1990.

15. S. Goldwasser and S. Micali. Probabilistic encryption. Journal of Computer and System Sciences, 28:270-299, 1984.

16. M. Hirt and U. Maurer. Player simulation and general adversary structures in perfect multiparty computation. Journal of Cryptology, 13(1):31-60, 2000.

17. C. A. R. Hoare. Communicating Sequential Processes. International Series in Computer Science, Prentice Hall, Hemel Hempstead, 1985.

18. D. M. Johnson and F. Javier Thayer. Security and the composition of machines. In Proc. 1st IEEE Computer Security Foundations Workshop (CSFW), pages 72-89, 1988.

19. P. Lincoln, J. Mitchell, M. Mitchell, and A. Scedrov. A probabilistic poly-time framework for protocol analysis. In Proc. 5th ACM Conference on Computer and Communications Security, pages 112-121, 1998.

20. P. Lincoln, J. Mitchell, M. Mitchell, and A. Scedrov. Probabilistic polynomial-time equivalence and security analysis. In Proc. 8th Symposium on Formal Methods Europe (FME 1999), volume 1708 of $L N C S$, pages 776-793. Springer, 1999. 
21. N. Lynch. Distributed Algorithms. Morgan Kaufmann Publishers, San Francisco, 1996.

22. H. Mantel. On the composition of secure systems. In Proc. 23rd IEEE Symposium on Security \& Privacy, pages 88-101, 2002.

23. D. McCullough. Specifications for multi-level security and a hook-up property. In Proc. 8th IEEE Symposium on Security \& Privacy, pages 161-166, 1987.

24. D. McCullough. A hookup theorem for multilevel security. IEEE Transactions on Software Engineering, 16(6):563-568, 1990.

25. J. McLean. A general theory of composition for trace sets closed under selective interleaving functions. In Proc. 15th IEEE Symposium on Security \& Privacy, pages 79-93, 1994.

26. J. McLean. A general theory of composition for a class of "possibilistic" security properties. IEEE Transactions on Software Engineering, 22(1):53-67, 1996.

27. S. Micali and P. Rogaway. Secure computation. In Advances in Cryptology: CRYPTO '91, volume 576 of LNCS, pages 392-404. Springer, 1991.

28. J. Misra and K. M. Chandy. Proofs of network of processes. IEEE Transactions on Software Engineering, 7(4):417-426, 1981.

29. B. Pfitzmann and M. Waidner. A general framework for formal notions of "secure" systems. Research Report 11/94, University of Hildesheim, Apr. 1994. http://www.semper.org/sirene/lit/abstr94.html\\#PfWa_94.

30. B. Pfitzmann and M. Waidner. Composition and integrity preservation of secure reactive systems. In Proc. 7th ACM Conference on Computer and Communications Security, pages 245-254, 2000. Extended version (with Matthias Schunter) IBM Research Report RZ 3206, May 2000, http://www . semper . org/sirene/publ/PfSW1_00ReactSimulIBM.ps.gz.

31. B. Pfitzmann and M. Waidner. A model for asynchronous reactive systems and its application to secure message transmission. In Proc. 22nd IEEE Symposium on Security \& Privacy, pages 184-200, 2001. Extended version in Cryptology ePrint Archive, Report 2000/066, http://eprint.iacr.org/

32. J. Widom, D. Gries, and F. B. Schneider. Trace-based network proof systems: Expressiveness and completeness. ACM Transactions on Programming Languages and Systems, 14(3):396416, 1992.

33. A. C. Yao. Protocols for secure computations. In Proc. 23rd IEEE Symposium on Foundations of Computer Science (FOCS), pages 160-164, 1982.

34. A. C. Yao. Theory and applications of trapdoor functions. In Proc. 23rd IEEE Symposium on Foundations of Computer Science (FOCS), pages 80-91, 1982. 